\title{
Concordancia entre escalas Framingham ATP III, SCORE y ACCIAHA 2013 \\ En una cohorte de pacientes en un hospital de cuarto nivel en el año 2015*
}

\author{
Concordance between the Framingham ATP III \\ SCORE and ACC/AHA 2013 Scales \\ En a cohort of patients in a fourth level hospital in 2015
}

\author{
Pedro Mancera-Rincón, Helman Eduardo Giral-Giraldo, Víctor Zein Rizo-Tello, \\ Édgar Camilo Barrera-Garavito • Bogotá, D.C. (Colombia)
}

\section{Resumen}

Introducción: identificar las personas sin enfermedad cardiovascular establecida con alto riesgo es el objetivo de la intervención primaria en prevención cardiovascular. En nuestro medio se han aplicado múltiples escalas de riesgo cardiovascular; sin embargo, en Colombia la única escala validada es la de Framingham.

Objetivo: estimar la concordancia entre las escalas Framingham ATP III, SCORE y ACC/ AHA 2013 para la predicción de riesgo cardiovascular en pacientes entre 40 y 75 años en una institución de cuarto nivel durante el año 2015.

Material y métodos: estudio de tipo observacional de corte transversal en pacientes de 40 75 años que asistieron durante el año 2015 en el servicio de chequeo general de un hospital de cuarto nivel, de 4783 individuos se tomó una muestra aleatorizada simple de 861, se calculó el riesgo cardiovascular con las escalas de Framingham, SCORE y AHA/ACC2013. Se describieron las variables cualitativas mediante distribuciones de frecuencias, las variables cuantitativas con medidas de tendencia central, se realizó un análisis bivariado con el coeficiente de correlación Kappa de Cohen considerándose como buena correlación $>60$.

Resultados: el cálculo del riesgo cardiovascular con cada una de las escalas encontró para alto riesgo AHA 2013 de 14.6\%, Framingham 2.2\% y SCORE con 1.1\%. Para riesgo medio SCORE de $26.9 \%$, AHA 2013 de $17.1 \%$ y Framingham de $14.4 \%$, y riesgo bajo la estimación fue de Framingham de $83.3 \%$, SCORE de 73\% y AHA 2013 68.3\%. El índice de concordancia de Kappa de Cohen para alto riesgo cardiovascular entre la escala Framingham modificada y SCORE se evidencia fuerza de concordancia moderada (Kappa: 0.47) al calcular este índice entre Framingham modificada y AHA 2013 la fuerza concordancia es débil (Kappa: 0.3497).

Conclusión: con los hallazgos del estudio se concluye que el comportamiento en cuanto a la estimación de riesgo de las escalas de SCORE y AHA 2013 no es concordante, por lo tanto, sus estimaciones no son intercambiables, tendiendo a sobreestimar o subestimar el riesgo. (Acta Med Colomb 2018; 43: 192-199).

Palabras clave: estudio, concordancia, riesgo, cardiovacular.

\section{Abstract}

Introduction: identifying people without established cardiovascular disease at high risk is the goal of primary intervention in cardiovascular prevention. In our environment, multiple scales of cardiovascular risk have been applied; however, in Colombia the only scale validated is Framingham.

Objective: to estimate the agreement between the Framingham ATP III, SCORE and ACC / AHA 2013 scales for the prediction of cardiovascular risk in patients between 40 and 75 years old in a fourth level institution during 2015.
*Trabajo Ganador del Concurso en la Modalidad de "Trabajo de Investigación Residentes", presentado durante el XXV Congreso Colombiano de Medicina Interna, 16 - 18 de agosto de 2018, Cali (Colombia).

Dres. Pedro Mancera-Rincón, Helman Eduardo Giral-Giraldo, Víctor Zein Rizo-Tello, Édgar Camilo Barrera-Garavito: Fundación Cardioinfantil, Instituto de Cardiología, Bogotá, D.C. (Colombia).

Correspondencia. Dr. Helman Eduardo GiralGiraldo. Bogotá, D.C. (Colombia). E-mail: helman.giral@gmail.com Recibido: 30/V/2018 Aceptado: 17/VIII/2018 
Material and methods: cross-sectional observational study in patients aged 40-75 years who attended in 2015 in the general check-up service of a fourth-level hospital. Of 4783 individuals a simple randomized sample of 861 was taken. Cardiovascular risk with the Framingham, SCORE and AHA / ACC2013 scales was calculated. Qualitative variables were described by frequency distributions, quantitative variables with measures of central tendency, and a bivariate analysis was performed with Cohen's Kappa correlation coefficient considering as a good correlation $>60$.

Results: the calculation of cardiovascular risk with each of the scales found for high risk AHA 2013 of $14.6 \%$, Framingham $2.2 \%$ and SCORE with $1.1 \%$. For average risk SCORE of $26.9 \%$, AHA 2013 of $17.1 \%$ and Framingham of $14.4 \%$, and risk under the estimate was of Framingham of $83.3 \%$, SCORE of $73 \%$ and AHA $201368.3 \%$. The Cohen's Kappa concordance index for high cardiovascular risk between the modified Framingham scale and SCORE evidence a moderate concordance strength (Kappa: 0.47); when calculating this index between modified Framingham and AHA 2013 concordance strength is weak (Kappa: 0.3497).

Conclusion: with the findings of the study it is concluded that the behavior regarding the risk estimation of SCORE and AHA 2013 scales is not concordant; therefore, their estimates are not interchangeable, tending to overestimate or underestimate the risk. (Acta Med Colomb 2018; 43: 192-199).

Keywords: study, concordance, risk, cardiovacular

\section{Introducción}

La enfermedad cardiovascular es la principal causa de muerte a nivel mundial, con mayor prevalencia en países de ingresos bajos y medios (4). En Colombia, el Instituto Nacional de Salud (INS) (2013) en su informe de las principales causas de muerte en el país durante el periodo 1998-2011 encontró que 628630 muertes se relacionaron con la enfermedad cardiovascular (cardiopatía isquémica, enfermedad cerebrovascular, cardiopatía hipertensiva y cardiopatía reumática crónica), representando el $23.5 \%$ de todas las muertes. De éstas 628630 muertes el 56.3\% se debieron a cardiopatía isquémica, $30.6 \%$ a enfermedad cerebrovascular, $12.4 \%$ a cardiopatía hipertensiva y $0.5 \%$ a cardiopatía reumática crónica (5). La mayoría de estas patologías están relacionadas a factores de riesgo potencialmente modificables como lo son el tabaquismo, la dislipidemia, la hipertensión arterial, diabetes mellitus tipo 2, obesidad abdominal, consumo de alcohol y sedentarismo. A partir de esto se han desarrollado diferentes estrategias de intervención tanto farmacológicas y no farmacológicas para población con enfermedad cardiovascular establecida y la aparentemente sana en fases preclínicas, teniendo en cuenta que un pilar fundamental es la prevención. Actualmente se cuentan distintas escalas predictoras y de clasificadoras de riesgo cardiovascular en población aparentemente sana, pero sólo una está validada para usarse en nuestro medio, la de Framingham ATP III (6), recomendada para la estimación del riesgo cardiovascular según las guías colombianas para manejo de dislipidemias (7) . Existen otras escalas ampliamente conocidas y representativas de sociedades científicas como lo son SCORE (8) y AHA/ ACC 2013 (3).

Al comparar la estimación de riesgo cardiovascular sobre un mismo grupo poblacional y compararlo con los eventos cardiovasculares presentados a 10 años el estudio de Kavousi (2014) (9) encontró que la discriminación de la escala ACC/AHA tuvo una C estadística de 0.67 (95\% CI, 0.63-0.71) para hombres y 0.68 (95\% CI, 0.64-0.73) para mujeres con eventos cardiovasculares, para ATP III la discriminación C estadística fue 0.67 (95\% CI, 0.62- 0.72) para hombres y 0.69 (95\% CI, 0.63-0.75) para mujeres con eventos fuertes de enfermedad cardiaca, para SCORE la C estadística fue 0.76 (95\% CI, 0.70-0.82) para hombres y 0.77 (95\% CI, 0.71-0.83) en mujeres con muerte por enfermedad cardiovascular, sumado a la recomendación para uso de estatinas, ACC/AHA(2013) sugiere que en casi todos los hombres y en $65 \%$ de las mujeres mayores de 55 años se recomienda tratamiento médico, para las guías ATP III 52\% de los hombres y 35\% de las mujeres son recomendado para tratamiento médico, la guía ESC $66 \%$ de los hombres y $39 \%$ de las mujeres son recomendadas para tratamiento médico. Estas diferencias tanto en la recomendación para el manejo farmacológico como en el poder de discriminación de cada escala generan interrogantes sobre el comportamiento de dichas escalas en nuestro medio.

En el estudio el estudio de Muñoz, O. (6) evaluó la concordancia entre las escalas Framingham SCORE y ACC/AHA 2013 en un grupo poblacional de 800 personas provenientes de la clínica de prevención primaria del Hospital Militar Central en Bogotá (Colombia) durante los años 1984 y 1996, encontrando niveles de concordancia bajos entre SCORE países de bajo riesgo y Framingham ajustado para alto riesgo cardiovascular Kappa $=0.2808$ (IC 95\% 0,2519-0.3097) y concordancia moderada entre AHA /ACC 2013 y Framingham ajustado para la determinación de manejo farmacológico y no manejo Kappa $=0.5735$ (IC 95\% 0.5388-0.6082). 
Debido a los niveles de concordancia moderada frente a la recomendación de manejo farmacológico se planteó realizar el ejercicio de concordancia entre las tres escalas mencionadas en un grupo poblacional más próximo al tiempo actual y describir las características del grupo poblacional a estudio. Considerando una concordancia clínicamente importante para Kappa: 0.6, esto a raíz de que en los trabajos realizados previamente, los índices de concordancia más altos fueron al comparar Framingham y ACC/AHA 2013 con Kappa $=0.5735$ al comparar la indicación farmacológica (6) y FraminghamD'Agostino y SCORE con Kappa : 0.52 para alto riesgo cardiovascular (10).

\section{Material y métodos}

Estudio de tipo observacional de corte transversal en pacientes de 40 - 75 años que asistieron durante el año 2015 en el servicio de chequeo general de un hospital de cuarto nivel. El protocolo fue aprobado por los comités de investigación de la institución. Se realizó muestreo aleatorio simple de tipo probabilístico. Se contó con acceso a la base de datos del programa en un archivo Excel que contiene el total de la población atendida en el año 2015, que corresponde 4783 pacientes, la muestra se calculó con el software tamaño de muestra versión 1.1 Editorial Panamericana (26). Se tuvo en cuenta el tipo de análisis que se quiere realizar que es de concordancia mediante el índice de Kappa. Se consideró concordancia clínicamente importante para Kappa $\geq 0.6$, esto a raíz de que en los trabajos realizados previamente de concordancia, los índices más altos fueron al comparar Framingham y ACC/AHA
2013 con Kappa $=0.5735$ al comparar la indicación farmacológica (6) y Framingham-D'Agostino y SCORE con Kappa: 0.52 para alto riesgo cardiovascular (10). Con un error máximo permitido de 5\% una confianza de $95 \%$ un poder estadístico de 80 y estimando la concordancia con el índice kappa de 50\%, es decir desconocido, el tamaño de muestra necesario fue de 861 sujetos. Con un ajuste por pérdidas estimado del $50 \%$, debido al grupo de sujetos que no cumplian los criterios de inclusion encontrados en una revision previa, la muestra fue de 1291 sujetos.

La base de datos se elaboró en Excel 2013 y se procesó en el programa Open epi versión 3.012013 de distribución gratuita y Stata 14 licenciado. Se describirán las variables cualitativas mediante distribuciones de frecuencias, las variables cuantitativas con medidas de tendencia central como la media y mediana, de dispersión como la desviación estándar, se documentó las medidas de posición, y se realizó un análisis bivariado de las estimaciones de riesgo cardiovascular según las aplicaciones de Framingham (27), AHA 2013 (28) y SCORE (29) con el coeficiente de correlación Kappa de Cohen considerándose como buena correlación $\geq 0.60$.

\section{Resultados}

Ingresaron al estudio un total de 863 pacientes contenidos en la base de datos de la lista durante el año 2015. De esta población el $65.7 \%$ eran hombres, el promedio de edad es de 49.7 años con DE: 6.8. La distribución de las características de los pacientes se presenta en la Tabla 1.

Se realizó el cálculo del riesgo cardiovascular para cada paciente con cada una de las escalas a estudio, al realizar

Tabla 1. Características de la población del estudio.

\begin{tabular}{|c|c|c|c|c|}
\hline Variable & $\%$ & IC95\% & Promedio & Desviación estándar \\
\hline \multirow[t]{2}{*}{ Sexo } & Hombre 65.7 & & & \\
\hline & Mujer 34.3 & & & \\
\hline \multirow[t]{2}{*}{ Edad } & Hombre & & 49.88 & 6.6 \\
\hline & Mujer & & 49.33 & 7.1 \\
\hline Peso $(\mathrm{Kg})$ & & & 76.06 & 14.42 \\
\hline Talla $(\mathrm{cm})$ & & & 169.81 & 9.85 \\
\hline IMC $\left(\mathrm{Kg} / \mathrm{m}^{2}\right)$ & & & 25.93 & 4.57 \\
\hline \multirow[t]{2}{*}{ Ant. HTA } & NO 87.6 & $85.27-89.68$ & & \\
\hline & SI 12.4 & $10.32-14.73$ & & \\
\hline \multirow[t]{2}{*}{ Ant. DM2 } & NO 97.8 & $96.65-98.63$ & & \\
\hline & SI 2.2 & $1.37-3.35$ & & \\
\hline \multirow[t]{2}{*}{ Ant. de consumo de cigarrillo } & NO 93.74 & $91.97-95.22$ & & \\
\hline & SI 6.25 & $4.78-8.02$ & & \\
\hline \multirow[t]{2}{*}{ Ant. de consumo de SPA } & NO 99.76 & $99.24-99.96$ & & \\
\hline & SI 0.23 & $0.03-0.76$ & & \\
\hline Colesterol total (mg/dL) & & & 211 & 37.76 \\
\hline Colesterol HDL (mg/dL) & & & 44.5 & 10.7 \\
\hline Colesterol LDL (mg/dL) & & & 138.8 & 32.95 \\
\hline
\end{tabular}


Tabla 2. Escala Framingham cruda.

\begin{tabular}{|l|c|c|c|}
\hline & Frecuencia & Porcentaje (\%) & Acumulado (\%) \\
\hline Alto & 50 & 5.79 & 5.79 \\
\hline Medio & 593 & 68.71 & 74.51 \\
\hline Bajo & 220 & 25.49 & 100.00 \\
\hline Total & 863 & 100 & \\
\hline
\end{tabular}

Tabla 3. Escala Framingham modificado.

\begin{tabular}{|l|c|c|c|}
\hline & Frecuencia & Porcentaje (\%) & Acumulado (\%) \\
\hline Alto & 19 & 2.20 & 2.20 \\
\hline Medio & 124 & 14.37 & 16.57 \\
\hline Bajo & 720 & 83.43 & 100.00 \\
\hline Total & 863 & 100 & \\
\hline
\end{tabular}

Tabla 4. Escala AHA 2013 (ASCVD).

\begin{tabular}{|l|c|c|c|}
\hline & Frecuencia & Porcentaje (\%) & Acumulado (\%) \\
\hline Alto & 126 & 14.60 & 14.60 \\
\hline Medio & 148 & 17.15 & 31.75 \\
\hline Bajo & 589 & 68.25 & 100.00 \\
\hline Total & 863 & 100 & \\
\hline
\end{tabular}

Tabla 5. Escala SCORE.

\begin{tabular}{|l|c|c|c|}
\hline & Frecuencia & Porcentaje (\%) & Acumulado (\%) \\
\hline Alto & 10 & 1.16 & 1.16 \\
\hline Bajo & 621 & 71.96 & 100.00 \\
\hline Total & 863 & 100 & \\
\hline
\end{tabular}

el análisis univariado estratificado se encontró que para la categoría alto riesgo la escala AHA 2013 estima la mayor cantidad de pacientes como alto riesgo (14.6\%) seguido de Framingham $2.2 \%$ y por último SCORE con $1.1 \%$. Cuando se realiza el análisis del riesgo medio SCORE estima $26.9 \%$ de la población seguido por AHA 2013 17.1\% Framingham $14.4 \%$ y para el estrato correspondiente a riesgo bajo la estimación es Framingham $83.3 \%$ seguido por SCORE 73\% y por último AHA 2013 68.3\% (Tablas 2-5).

Es importante tener en cuenta que la escala de estimación de riesgo cardiovascular validada para Colombia es Framingham, sin embargo, la recomendación realizada es que debe realizarse un ajuste en los datos multiplicando el valor calculado que se encentra validado por 0.75 . Cuando se comparan los datos estimados con la escala Framingham crudo con los de la modificada se evidencia que los casos clasificados en la modificada disminuyen en los niveles alto y bajo y aumenta la captación de pacientes con riesgo intermedio (Tablas 2 y 3 ).

Para efectos de esta investigación se usó la escala Framingham modificada como patrón de oro contra la cual se realizaron las comparaciones y los cálculos de análisis de sensibilidad y correlación.

Se realizó un análisis bivariado en Tablas 2 x 2, en donde los valores son las concordancias de pacientes que son positivos para alto riesgo en ambas escalas, esto con el fin de evaluar el rendimiento de las mismas. La prueba SCORE sólo detecta $26 \%$ de los pacientes de alto riesgo, pero tiene buena especificidad detectando así $99.4 \%$ de los que son de bajo y moderado riesgo, con un muy buen valor predictivo negativo y precisión diagnóstica. En cuanto a la concordancia, presenta un nivel de acuerdo leve con un Kappa $=0.335($ IC $0.039-0.631)($ Tabla 7) .

Tabla 6. Tabla de frecuencias Framingham modificado alto riesgo Vs AHA 2013 alto riesgo.

\begin{tabular}{|c|c|c|c|c|}
\hline \multirow{5}{*}{ AHA 2013} & \multicolumn{4}{|c|}{ Framingham modificado } \\
\hline & & Positivo & Negativo & Total \\
\hline & Positivo & 19 & 107 & 126 \\
\hline & Negativo & 0 & 737 & 737 \\
\hline & Total & 19 & 844 & 863 \\
\hline \multicolumn{2}{|c|}{ Parámetro } & Cálculo & IC $95 \%$ Inferior- Superior & Método \\
\hline \multicolumn{2}{|l|}{ Sensibilidad } & $100 \%$ & $(83.18,1001)$ & Puntos de Wilson \\
\hline \multicolumn{2}{|l|}{ Especificidad } & $87.32 \%$ & $(84.91,89.41)$ & Puntos de Wilson \\
\hline \multicolumn{2}{|c|}{ Valor predictivo positivo } & $15.08 \%$ & $(9.871,22.351)$ & Puntos de Wilson \\
\hline \multicolumn{2}{|c|}{ Valor predictivo negativo } & $100 \%$ & $(99.48,1001)$ & Puntos de Wilson \\
\hline \multicolumn{2}{|c|}{ Precisión de diagnóstico } & $87.6 \%$ & $(85.23,89.641)$ & Puntos de Wilson \\
\hline \multicolumn{2}{|c|}{ Razón de verosimilitud de prueba positiva } & 7.888 & $(7.745-8.034)$ & \\
\hline \multicolumn{2}{|c|}{ Razón de verosimilitud de prueba negativa } & $\begin{array}{c}0.0 \\
\text { No definido }\end{array}$ & $(0.0-)$ & \\
\hline \multicolumn{2}{|l|}{ Índice Kappa } & 0.233 & $(0.097-0.369)$ & \\
\hline
\end{tabular}


Al realizar el análisis estratificado de sensibilidad de alto riesgo se evidencia que AHA 2013 detecta el total de pacientes de alto riesgo comparados con el gold estándar y detecta $87 \%$ de los de moderado y bajo riesgo. En cuanto a la concordancia entre Framingham y AHA 2013 el nivel de acuerdo es leve con un Kappa $=0.233$ (IC 0.097-0.369) (Tabla 6).

El análisis de prueba realizado a la escala SCORE, muestra un bajo rendimiento para indicar manejo farmacológico en comparación con Framingham modificado con una sensibilidad del $50 \%$ pero con un mejor rendimiento a la hora de determinar quienes no se benefician de manejo farmacológico con una especificidad de $96.3 \%$; el nivel de concordancia es moderado con un Kappa $=0.5282$ (IC $0.4651-0.5914)$ (Tabla 7).

Al analizar el rendimiento de la escala AHA 2013, se evidencia una mala sensibilidad para indicar manejo farmacológico con una sensibilidad de $50.36 \%$ pero una mejor capacidad de discriminar a quienes no tienen indicación farmacológica (especificidad 98.98\%). El nivel de concordancia es moderado con un Kappa $=0.5652$ (IC $0.504-0.6264$ ) (Tabla 9).

En el presente estudio realizado en la población de Chequeo ejecutivo durante el año 2015 en pacientes sin enfermedad cardiovascular establecida entre los 40 y 75 años de edad, se aplicaron tres escalas de riesgo cardiovascular;

Tabla 7. Tabla de frecuencias Framingham Modificado Alto riesgo Vs SCORE alto riesgo.

\begin{tabular}{|c|c|c|c|c|}
\hline & \multicolumn{4}{|c|}{ Framingham modificado } \\
\hline \multirow{2}{*}{ SCORE } & Negativo & 14 & 839 & 853 \\
\hline & Total & 19 & 844 & 863 \\
\hline \multicolumn{2}{|c|}{ Parámetro } & Cálculo & IC $95 \%$ Inferior- Superior & Método \\
\hline \multicolumn{2}{|l|}{ Sensibilidad } & $26.32 \%$ & $(11.81,48.79)$ & Puntos de Wilson \\
\hline \multicolumn{2}{|l|}{ Especificidad } & $99.41 \%$ & $(98.62,99.75)$ & Puntos de Wilson \\
\hline \multicolumn{2}{|c|}{ Valor predictivo positivo } & $50 \%$ & $(23.66,76.34)$ & Puntos de Wilson \\
\hline \multicolumn{2}{|c|}{ Razón de verosimilitud de prueba negativa } & $\begin{array}{c}0.7412 \\
59.93\end{array}$ & $\begin{array}{c}(0.6444-0.8526) \\
(15.58-230.6)\end{array}$ & \\
\hline \multicolumn{2}{|l|}{ Índice Kappa } & 0.335 & $(0.039-0.631)$ & \\
\hline
\end{tabular}

Tabla 8. Tabla de frecuencias para Framingham modificado Vs SCORE según indicación farmacológica.

\begin{tabular}{|l|l|c|c|c|}
\hline \multirow{5}{*}{ SCORE } & \multicolumn{4}{|c|}{ Framingham modificado } \\
\hline & & Positivo & Negativo & Total \\
\cline { 2 - 6 } & Positivo & 121 & 23 & 144 \\
\cline { 2 - 6 } & Negativo & 121 & 598 & 719 \\
\cline { 2 - 6 } & Total & 242 & 621 & 863 \\
\hline
\end{tabular}

\begin{tabular}{|l|c|c|c|}
\hline \multicolumn{1}{|c|}{ Parámetro } & Calculo & IC 95\% Inferior - Superior & Método \\
\hline Sensibilidad & $50 \%$ & $(43.75,56.25)$ & Puntos de Wilson \\
\hline Especificidad & $96.3 \%$ & $(94.5,97.52)$ & Puntos de Wilson \\
\hline Valor predictivo positivo & $84.03 \%$ & $(77.17,89.11)$ & Puntos de Wilson \\
\hline Valor predictivo negativo & $83.17 \%$ & $(80.26,85.73)$ & Puntos de Wilson \\
\hline Precisión de diagnóstico & $83.31 \%$ & $(80.68,85.65)$ & Puntos de Wilson \\
\hline Razón de verosimilitud de prueba positiva & 13.5 & $(12.2-14.94)$ & $(0.5108-0.5278)$ \\
\hline Razón de verosimilitud de prueba negativa & 0.5192 & $(15.98-42.3)$ & $(0.4651-0.5914)$ \\
\hline Kappa de Cohen & 26 & 0.5282 & \\
\hline
\end{tabular}


Tabla 9. Tabla de frecuencias para Framingham modificado Vs AHA según indicación farmacológica.

\begin{tabular}{|c|c|c|c|c|}
\hline & \multicolumn{4}{|c|}{ AHA 2013} \\
\hline \multirow{3}{*}{ Framingham } & & Positivo & Negativo & Total \\
\hline & Negativo & 139 & 583 & 719 \\
\hline & Total & 274 & 589 & 863 \\
\hline
\end{tabular}

\begin{tabular}{|l|c|c|c|}
\hline \multicolumn{1}{|c|}{ Parámetro } & Calculo & IC 95\% Inferior - Superior & Método \\
\hline Sensibilidad & $50.36 \%$ & $(44.48,56.24)$ & Puntos de Wilson \\
\hline Especificidad & $98.98 \%$ & $(97.8,99.53)$ & Puntos de Wilson \\
\hline Valor predictivo positivo & $95.83 \%$ & $(91.21,98.08)$ & Puntos de Wilson \\
\hline Valor predictivo negativo & $81.08 \%$ & $(78.08,83.78)$ & Puntos de Wilson \\
\hline Precisión de diagnóstico & $83.55 \%$ & $(80.92,85.87)$ & Puntos de Wilson \\
\hline Razón de verosimilitud de prueba positiva & 49.44 & $(35.17-69.51)$ & $(0.504-0.6264)$ \\
\hline Razón de verosimilitud de prueba negativa & 0.5652 & $(42.63-228)$ \\
\hline Kappa de Cohen & 98.6 & $(0.504-0.6264)$ & \\
\hline
\end{tabular}

Framingham modificado (7), AHA/ACC 2013 (3) y SCO$\mathrm{RE}$ (8), identificando en los 863 pacientes los altos riesgos, definidos por Framingham > 20\%, AHA/ACC $2013>7.5 \%$ y SCORE $>5 \%$, representaron un $2.20 \%, 14.60 \%$ y $1.16 \%$ de toda la población respectivamente. Al evaluar el grado de concordancia global de las escalas y asumiendo como "gold standard" la escala de Framingham modificada; se encontró concordancia moderada entre Framingham modificado y SCORE $($ Kappa $=0.4765)$, concordancia débil entre Framingham y AHA/ACC 2013 (Kappa=0.3497). De igual forma el nivel de concordancia para estraficación de alto riesgo fue débil entre Framingham y SCORE países de bajo riesgo $($ Kappa $=0.335)$ y Framingham y AHA/ACC $2013($ Kappa $=0.233)$, en cuanto a la concordancia entre la indicación de manejo farmacológico, la concordancia fue algo mejor con un nivel moderado de acuerdo entre Framingham y SCORE países de bajo riesgo $($ Kappa $=$ 0.5282) al igual que entre Framingham y AHA/ACC 2013 $($ Kappa $=0.5652)$.

La estimación del riesgo cardiovascular presenta muchos limitantes, el principal es la carencia de un verdadero gold standard, diferentes modelos predictivos se han desarrollado, los más ampliamente conocidos en nuestros medios son los citados en este estudio. La escala Framingham, que fue retomada en el ATP III (1), entiende por enfermedad cardiovascular a la probabilidad de muerte por enfermedad coronaria cardiaca e infarto no fatal, diferente a lo que SCORE identifica, esta escala predice el riesgo de enfermedad cardiovascular fatal a 10 años, muy distante éstas dos a lo que AHA/ACC 2013 definió como enfermedad cardiovascular, que fue la probabilidad de presentar infarto agudo de miocardio no fatal, muerte por enfermedad cardiaca coronaria, accidente cerebrovascular isquémico fatal o no. Lo anterior refleja lo que cada escala entiende por enfermedad cardiovascular, de ahí que su estimación debe ser diferente, según lo que se busque prevenir. Ahora en Colombia solo contamos con la validación de escala Framingham (11), la cual demostró una sobreestimación de riesgo cardiovascular con una relación de eventos esperados y observados de 1.31 y describiendo una baja capacidad de discriminación (AUC 0.74). En la guía colombiana para manejo de dislipidemias, se retomó el uso de la escala Framigham con la precaución de ajustar el riesgo cardiovascular por 0.75 para corregir el posible efecto de sobreestimación (7), pero a pesar del ajuste y según referencias internacionales es probable que exista aun sobreestimación del riesgo.

\section{Discusión}

Los ejercicios de concordancia realizados previamente han demostrado a nivel de Europa una concordancia moderada a débil entre Framingham y SCORE, que han sido las escalas más utilizadas, ejemplo de esto el estudio de Ruiz-Villaverde (30) en pacientes con síndrome metabólico, con una concordancia débil para alto riesgo cardiovascular (Kappa=0.224), Gil Guillen en 2007 (23) comparó SCORE y REGICOR- Framingham en población sin enfermedad cardiovascular encontrando correlación moderada (Kappa $=0.4$ ) para alto riesgo cardiovascular. En Latinoamérica Bazo Álvarez en el 2015 (24) compararon AHA/ACC 2013 y otras seis escalas ( Framingham, Framingham sin laboratorio, Reynolds, SCORE, World Health Organization risk charts y Lancet chronic diseases risk charts) encontraron una pobre correlación (Índice de Lin`s AHA/ACC 2013 Vs Framingham $=0.44$, AHA/ACC 2013 Vs SCORE = 0.14), en México Alcocer, L. (25) documentó 
una concordancia del $3 \%$ para alto riesgo cardiovascular. En Colombia el estudio de Muñoz O. (6) evaluó un grupo poblacional de 800 personas provenientes de la clínica de prevención primaria del Hospital Militar Central en Bogotá durante los años 1984 y 1996, estimó el riesgo cardiovascular con las escalas de Framingham, SCORE y ACC/AHA 2013 encontrando niveles de concordancia débiles para alto riesgo cardiovascular entre SCORE países de bajo riesgo y Framingham ajustado (Kappa $=0,2808)$ que son similares a los encontrados en nuestro estudio (Kappa=0.335), al comparar AHA /ACC 2013 y Framingham ajustado para indicación farmacológica encontraron concordancia moderada (Kappa: 0.5735) que son similares a los datos encontrados en nuestro estudio (Kappa: 0.5652).

La principal limitación de este estudio es su carácter transversal sin seguimiento, adecuado para la evaluación de concordancia entre escalas. La muestra poblacional no cumple las características representativas de población colombiana y refleja al grupo poblacional del programa de chequeo ejecutivo de FCI por lo que podría haber variación de los resultados en diferentes regiones del país. Los resultados sugieren que la escala AHA/ACC 2013 podría sobreestimar el riesgo cardiovascular al igual que previamente se registró en nuestro país con la escala Framingham, que aunque modificada, sigue siendo una escala con un poder de discriminación subóptimo, por lo que se plantea que una escala como SCORE para países de bajo riesgo cardiovascular pueda tener una estimación diferente con tendencia a una menor sobreestimación en nuestra población, por lo que sería necesario realizar estudios observacionales con seguimiento a 10 años para determinar el nivel de precisión y capacidad de discriminación de esta escala.

\section{Conclusiones}

Según los resultados presentados, concluimos que la estimación de riesgo cardiovascular e indicación farmacológica entre las escalas de Framingham modificado, AHA/ ACC 2013 y SCORE no son concordantes entre sí; esto plantea una peor o mejor estimación de riesgo para AHA/ ACC 2013 y SCORE en comparación con Framingham.

La escala AHA/ACC 2013 presentó un mayor porcentaje de estimación de paciente en alto riesgo cardiovascular; lo que podría estar relacionado a un posible efecto de sobreestimación de riesgo, por lo que se sugiere a futuro validar nuevas escalas en nuestra población, especialmente escalas que presenten un menor riesgo de sobre estimación de riesgo.

\section{Referencias}

1. Program T report of the national cholesterol education. Final Report. Transportation (Amst). 2002;02(5215).

2. Reinereljko, Catapano AL, De Backer G, Graham I, Taskinen MR, Wiklund O, et al. ESC/EAS Guidelines for the management of dyslipidaemias. Eur Heart J. 2011; 32(14): 1769-818.

3. Ray KK, Kastelein JJP, Matthijs Boekholdt S, Nicholls SJ, Khaw KT, Ballantyne CM, et al. The ACC/AHA 2013 guideline on the treatment of blood cholesterol to reduce atherosclerotic cardiovascular disease risk in adults: The good the bad and the uncertain: A comparison with ESC/EAS guidelines for the management of dyslipidaemias 2011. Eur Heart J. 2014; 35(15): 960-8.

4. Organización Mundial de la Salud. Informe sobre la situación mundial de las enfermedades no transmisibles. WHO. 2014;1-18.

5. Instituto Nacional de Salud. Enfermedad cardiovascular : principal causa de muerte en Colombia. Boletín ONS [Internet]. 2013;(1):1-6. Disponible en: http://www.ins.gov.co/lineas-de-accion/ons/boletin 1/boletin_web_ONS/ boletin_01_ONS.pdf

6. Muñoz V OM, Ruiz Morales ÁJ, Mariño Correa A, Bustos C. MM. Concordancia entre los modelos de SCORE y Framingham y las ecuaciones AHA/ACC como evaluadores de riesgo cardiovascular. Rev Colomb Cardiol. 2016;(xx).

7. Adulto CDEL, Merchán A, Sánchez G. Guía de práctica clínica para la prevención, detección temprana, diagnóstico, tratamiento y seguimiento de las dislipidemias: tratamiento farmacológico con estatinas. 2015; 22(1): 14-21.

8. Conroy RM, Pyörälä K, Fitzgerald AP, Sans S, Menotti A, De Backer G, et al. Estimation of ten-year risk of fatal cardiovascular disease in Europe: The SCORE project. Eur Heart J. 2003;24(11):987-1003.

9. Kavousi M, Leening MJG, Nanchen D, Greenland P, Graham IM, Steyerberg EW, et al. Comparison of application of the ACC/AHA guidelines, Adult Treatment Panel III guidelines, and European Society of Cardiology guidelines for cardiovascular disease prevention in a European cohort. JAMA [Internet]. 2014;311(14):1416-23. Disponible en: http://www.ncbi.nlm.nih.gov/ pubmed/24681960

10. Gomez-Marcos MA, Grandes G, Iglesias-Valiente JA, Sánchez A, Montoya I, Garcia-Ortiz L, et al. Agreement between the SCORE and D'Agostino scales for the classification of high cardiovascular risk in sedentary Spanish patients. Int J Environ Res Public Health. 2009;6(11):2800-11.

11. Muñoz OM, Rodríguez NI, Ruiz Á, Rondón M. Validación de los modelos de predicción de Framingham y PROCAM como estimadores del riesgo cardiovascular en una población colombiana. Rev Colomb Cardiol [Internet]. 2014; 21(4): 202-12. Disponible en: http://www.sciencedirect.com/science/article/pii/ S0120563314000126

12.Data GHO (GHO). Top 10 causes of death [Internet]. 2015. Disponible en: http://www.who.int/gho/mortality_burden_disease/causes_death/top_10/en/

13. Yusuf S, Hawken S, Ôunpuu S, Dans T, Avezum A, Lanas F, et al. Effect of potentially modifiable risk factors associated with myocardial infarction in $\mathbf{5 2}$ countries in a case-control study based on the INTERHEART study. Lancet. 2004; 364: 937-52.

14. Wilson PWF, D'Agostino RB, Levy D, Belanger AM, Silbershatz H, Kannel WB. Prediction of Coronary Heart Disease Using Risk Factor Categories. Circulation [Internet]. 1998; 97(18): 1837-47. Disponible en: http://circ.ahajournals. org/cgi/doi/10.1161/01.CIR.97.18.1837.

15. Kannel WB, McGee D, Gordon T. A general cardiovascular risk profile: The Framingham study. Am J Cardiol. 1976; 38(1): 46-51.

16. Sullivan LM, Wilson P. Validation of the Framingham Coronary Heart Disease prediction score. JAMA. 2001;286(2):180-7.

17. The atherosclerosis risk in communities (ARIC) study: design and objectives Am J Epidemiol [Internet]. el 1 de abril de 1989;129(4):687-702. Disponible en: https://academic.oup.com/aje/article-lookup/doi/10.1093/oxfordjournals aje.a115184

18. Fried LP, Borhani NO, Enright P, Furberg CD, Gardin JM, Kronmal R a, et al. The Cardiovascular Health Study: design and rationale. Ann Epidemiol [Internet]. 1991; 1(3): 263-76. Disponible en: http://www.ncbi.nlm.nih.gov/ pubmed/1669507

19. Manolio TA, Savage PJ, Burke GL, Liu KA, Wagenknecht LE, Sidney S, et al. Association of fasting insulin with blood pressure and lipids in young adults. The CARDIA study. Arteriosclerosis [Internet]. 1990;10(3):430-6. Disponible en: http://www.ncbi.nlm.nih.gov/pubmed/2188641.

20. Bild DE, Bluemke DA, Burke GL, Detrano R, Diez Roux A V., Folsom AR, et al. Multi-Ethnic Study of Atherosclerosis: Objectives and design. Am J Epidemiol. 2002; 156(9): 871-81.

21. Catapano AL, Graham I, De Backer G, Wiklund O, Chapman MJ, Drexel H, et al. 2016 ESC/EAS Guidelines for the Management of Dyslipidaemias. Eur Heart J. 2016; 37(39): 2999-30581.

22.Paixao ARM, Ayers CR, Berry JD, De Lemos JA, Khera A. Atherosclerotic cardiovascular disease prevention: A comparison between the third adult treatment panel and the new 2013 treatment of blood cholesterol guidelines. Circ Cardiovasc Qual Outcomes. 2014; 7(5): 778-9.

23. Gil-Guillén V, Orozco-Beltrán D, Maiques-Galán A, Aznar-Vicente J, Navarro J, Cea-Calvo L, et al. Concordancia de las escalas REGICOR y SCORE 
para la identificación del riesgo cardiovascular alto en la población española ARTÍCULO ORIGINAL Agreement Between REGICOR and SCORE Scales in Identifying High Cardiovascular Risk in the Spanish Population. Rev Esp Cardiol. 2007; 60(10): 1042-50.

24.Bazo-alvarez JC, Quispe R, Peralta F, Poterico JA, Valle GA, Burroughs $\mathbf{M}$, et al. Agreement Between Cardiovascular Disease Risk Scores in ResourceLimited Settings : Evidence from 5 Peruvian Sites. Crit Pathways Cardiol 2015. 2015; 14(2): 74-80.

25. Alcocer LA, Lozada O, Fanghänel G, Sánchez-reyes L, Campos-franco E. en población mexicana del estudio PRIT. 2011;168-74.

26. Ruiz Morales ÁJ. Epidemiología Clínica: Investigación clínica aplicada. Segunda. Bogotá: Panamericana; 2015.639 p.

27. Wilson, D'Agostino $\mathbf{L}$ et al. Framingham Heart Study [Internet]. [citado el
10 de mayo de 2018]. Disponible en:https://www.framinghamheartstudy.org/ fhs-risk-functions/coronary-heart-disease-10-year-risk/

28. Whelton PK, Carey RM, Aronow WS, Casey DE, Collins KJ, Dennison Himmelfarb C, et al. ASCVD Risk Estimator Plus [Internet]. Vol. 71. J Am Col Card 2018 [citado el 10 de mayo de 2018]. p. e127-248. Disponible en: http://tools.acc.org/ascvd-risk-estimator-plus/\#!/calculate/estimate/

29. HeartScore - Login [Internet]. [citado el 10 de mayo de 2018]. Disponible en: https://heartscore.escardio.org/2007/Login.aspx?ReturnUrl=\%2F2007\%2Fdef ault.aspx\%3Fmodel\%3Dspain-castillan\&model=spain-castillan

30. Ruiz-Villaverde G, Sánchez-Cano D, Ruiz-Villaverde R, Ábalos-Medina GM, Ramírez-Rodrigo J, Villaverde-Gutiérrez C. Agreement between Framingham-DORICA and SCORE scales in estimation of cardiovascular risk in the patients suffering from metabolic syndrome in Granada (Spain). Ir J Med Sci. 2011; 180(2): 351-4. 